Usage, Usability, and Utility of 3D City Models, 03010 (2012)

DOI: $10.1051 / 3 \mathrm{u} 3 \mathrm{~d} / 201203010$

(C) Owned by the authors, published by EDP Sciences, 2012

\title{
3D spatial information infrastructure: The case of Port Rotterdam
}

\author{
S. Zlatanova ${ }^{1}$ and J. Beetz ${ }^{2}$ \\ 1 GIS Technology, OTB, Delft University of Technology, PO Box 5030, 2600 GA, Delft, \\ The Netherlands \\ 2 Design Systems, Faculty of Architecture, Eindhoven University of Technology, \\ Postbus 513, 5600 MB Eindhoven, The Netherlands
}

\begin{abstract}
The development and maintenance of the infrastructure, facilities, logistics and other assets of the Port of Rotterdam requires a broad spectrum of heterogeneous information. This information concerns features, which are spatially distributed above ground, underground, in the air and in the water. The data are managed in a variety of data models with varying levels of dimensionality, granularity, accuracy and upto-dateness. Additionally increasing number of 3D design models is becoming available. This complexity of tasks and diversity of information challenges the Port authority to look for more advanced 3D solutions. This paper presents research in progress related to developing a 3D SII in support of information and process management within the Port of Rotterdam.
\end{abstract}

\section{INTRODUCTION}

The Port of Rotterdam (www.portofrotterdam.com) is one of largest in the world and is the gateway to the European trade of more than 350 million consumers. The annual throughput is about 430 million tons. The Port is spread on the area of 105 sq. km over a distance of $45 \mathrm{~km}$. The construction of an extension of the harbour into the sea (Maasvlakte 2) has started in 2008, aiming at accommodating the first ship in 2013. As Maasvlakte 2 (www.maasvlakte2.com) gets operational, the industry will shift further to the sea and will open new possibilities for recreation and urban planning in the areas close to Rotterdam. Many of the harbours within the outlines of the city will change their major function. The harbour area accommodates a large number of companies among which the oil refineries, petrochemical industry and general cargo trans-shipment handlings are most prominent. Since 2007 the Betuweroute (www.betuweroute.nl), the fast cargo railway from Port of Rotterdam to Germany, has also become operational, which also contributes to the dynamic of the region. The area around the harbour is also densely populated.

The continuous development and maintenance of the infrastructure, facilities, logistics and other assets of the Port of Rotterdam requires the management of a broad spectrum of heterogeneous information. A large number of public and private stakeholders that include companies, environmental authorities, municipalities, various institutions and citizens are constantly involved in the exchange of critical information. Much of this information concerns interdependent infrastructural features that are embedded in a dynamic environment which is in a constant state of transformation. These features are spatially distributed above ground (topography, cadastral parcels, buildings, streets, parking areas), underground (cables and pipes, geological and geotechnical data, tunnels), in the air (sensors for measurement of air quality, radar coverage, camera coverage) and in the water (depth of the harbour). A variety of data models and data formats with varying levels of dimensionality, granularity, accuracy

This is an Open Access article distributed under the terms of the Creative Commons Attribution License 2.0, which permits unrestricted use, distribution, and reproduction in any medium, provided the original work is properly cited. 
and up-to-dateness are in use. There is an increasing awareness of the insufficiencies of information coverage, handling and exchange in the management of the Port of Rotterdam. Our investigations of processes and data within the Port identified several problem domains:

Low dimensionality: A substantial part of the data sets in the management and design processes of the Port consist of traditional 2D line drawings. For many applications such as the calculation of radar shadows by buildings, noise emission simulations, the distribution of emission from chimneys or the analysis of exact spatial configurations of complex layers of underground features (cables, pipes, tunnels, garages, underground) these flat representations need to be remodelled in their full three dimensional spatial extend.

High complexity of data sets. This complexity concerns several aspects. Geometric diversity: the variety of topographic and other thematic maps, high resolution measurement data from various sensors (optical, laser, water-lever sensors, etc.), voxel sets of representing geology and design information, provided by public and commercial services, results in large amounts of data which are challenging to process. Semantic diversity: for data based on existing semantically rich data models problems arise from matching and mapping different semantic concepts for an integration of heterogeneous data into a single model. Granularity \& accuracy: with varying geometric and topological detail of available data sets, abstraction, simplification and the provision of multiple levels of detail are necessary to facilitate the decision making process. The majority of data transferred between stakeholders and Port of Rotterdam is encoded in proprietary files (like shape, dwg, dgn) that require the use of a large range of specialized applications and tools with a high total cost of ownership/operation.

These aspects have been addresses by a nationally funded project 3DSDI for Port Rotterdam. This paper presents the motivation and goals of the project and elaborates on our ideas for an appropriate 3D Spatial Information Infrastructure (SII). The paper is organised as follows: the next section 2 briefly presents recent 3D developments, which can be used as basis for the intended 3D SII. Section 3 presents the scope of the intended 3D SII and the main research questions. Section 4 briefly discusses some initial results. Section 5 elaborates on the next steps and short coming developments.

\section{3D DEVELOPMENTS}

3D spatial information is becoming widely used in daily life. Large companies, small businesses as well as private persons have access to 3D spatial information (structured and non-structured) in various scales and resolutions and the need for integrated modelling (above, below and on the surface) is growing. The society is facing an important paradigm change of spatial information; from 2D to 3D [1-6]. This can be directly seen in the societies' growing interest for 3D geographic tools such as Google Earth, Virtual Earth, Second Life, GRIFINOR [7] etc. and also in the trend of development of all the large GIS manufactures.

The majority of the large companies dealing with spatial information have announced 3D functionality within database technology (Oracle Spatial 2007), Architecture Engineering and Construction (AEC) software (Bentley Systems, Autodesk,), GIS Software (ESRI) and spatial data processing (SAFE). CityGML was accepted as OGC 3D standard and upgraded recently to version 2.0 [8], Building Information Models (BIM) are becoming more mature and increasingly used for design ([9], Figure 1 right). Therefore the conversion between CityGML and BIM is becoming a hot topic of investigations [9-13]. The Dutch SDI organisation Geonovum is has made a crucial step towards 3D by developing the 3D standard for the large topographic map of the Netherlands on the basis of CityGML, i.e. 3DIMGeo ([6], Figure 1, left).

Semantics aspects of information are also increasingly in the focus of investigations. Numerous semantic mappings are reported in the literature $[14,15]$. This new technology also brings new challenges. The developments are very intensive, but due to differences in semantic, geometric and topological properties, no guarantees are given that the set of data from one GIS or CAD system can be seamlessly converted into another one. 


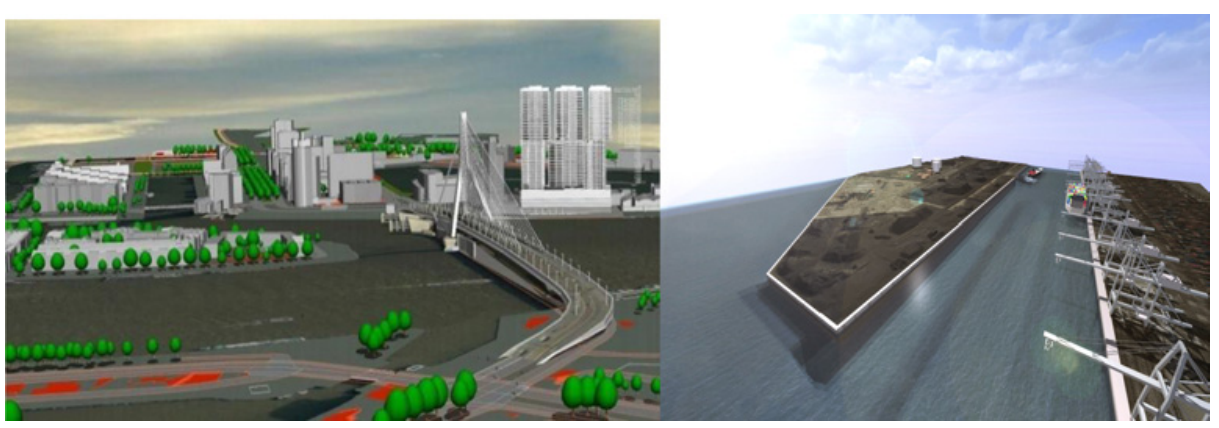

Figure 1. Availability of 3D information: 3D model of City of Rotterdam (left, courtesy Bentley Systems), and design model of the new quay, Port Rotterdam (right, courtesy City of Rotterdam).

All these developments establish a solid foundation for the development of 3D SII for such a huge company as Port Rotterdam. The numerous research and vendor developments have to be extensively investigated, analysed and compared with respect to the specific and challenges of the information management.

\section{3D SII FOR PORT ROTTERDAM}

3D SIIis expected to have a high positive impact for the Port in two major directions: 1) the internal and external information flow in Port of Rotterdam, and 3) the stakeholders of the entire region. On one hand, the improved models and system architectures shell increase the productivity and provide additional means to check the up-to-dateness, correctness and consistency of data. On the other hand, a system architecture that makes relevant information available on the internet will stimulate stakeholders to check and alert for inconsistencies and needed updates (i.e. 'citizens as sensors', [16, 17]). 3D SII is expected to prevent loss of critical data during the construction process (for renovation or new quays at Maasvlakte 2), will give better overview on the occupancy of industrial territories, infrastructure above and below the surface, keeping records about the geotechnical and geological conditions on land and sea shore. 3D measurements of sand excavations along the shore could be combined with the construction works within areas already in use. 3D models of the industrial areas can be later used for safety, security and surveillance or visibility analysis (e.g. for security cameras) replacing existing 2D maps.

Based on initial studies on process and data exchange, communication with clients and companies, and the needed data for management and maintenance of assets, we have identified that a generic 3D model (or set of models) has to be envisaged to allow integration of different data sets in one environment and for different department/client goals. This model should be seen as a major part of the SII and aims at the alignment, harmonization and integration of existing spatial data models as well as the development of additional umbrella meta-models and missing domain models. Related to the model, two aspects require special attention:

- The set of objects (features), which have to be included in the model considering their semantics, geometry, topology, appearance, granularity or levels of detail (LOD). Currently, one real-world phenomena might be represented in several department systems, having different attributes and, sometimes, different geometric representations. Going to 3D, it should be also evaluated which concept BIM (e.g. IFC) or GIS (e.g. CityGML) would be more relevant for the generic model.

- The data structure, which would be most appropriate to maintain the objects, their properties and relationships. At present many data sets are maintained by individual departments, although large parts of the information are managed centrally in database management system (DBMS). A central 


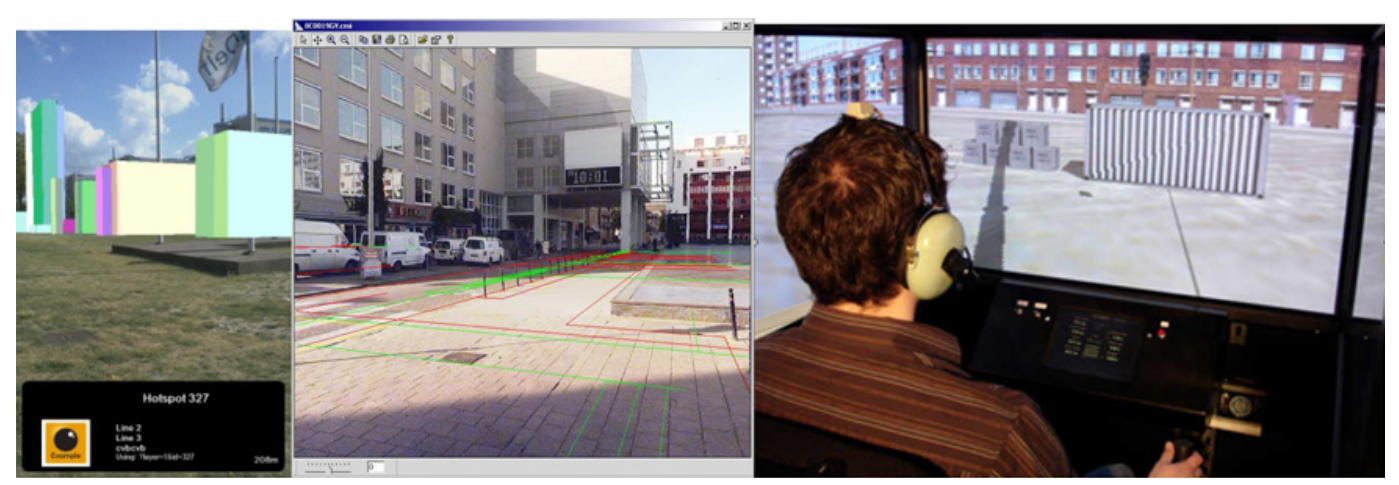

Figure 2. 3D visualisation of the TUD campus in Layar (left, courtesy Josafat Guerrero), overlay of utilities on panoramic images (middle, [18] and serious games (right, courtesy E-Semble).

management will facilitate consistency and re-use of information, but will require a data model that can serve the needs of all departments.

To replace the cumbersome and verbose exchange of file-based information between stakeholders, we intend to develop a Service Oriented Architecture that will allow just-in-time extraction and integration of distributed data sources through web services. We will reuse existing technologies such as the OGC family of geospatial information access standards through e.g. Web Feature Services (WFS) and developments in (spatial) queries of partial Building Information Models (BIM) such as the Information Delivery Manual (IDM) and Model View Definitions (MVD). On a technological level, mature, open and established technologies like SOAP, Google Protocol Buffers and RESTful services would guarantee their usability in production environments. Which approach will be followed depends on the types of uses and the tasks they need to perform. Related to this is the question of the standards, to be used for exchange of information: GML, CityGML, BIM (IFC) or the Dutch national standards from the series IMxxx.

To make efficient use of the structured, interconnected information provided by the model described earlier, data integration and visualization clients are developed. These allow the selection of relevant spatial data from different sources and provide visual means for multi-criteria and multi-dimensional analyses in $2 \mathrm{D}$ or $3 \mathrm{D}$ to support decision processes. Flexibility and ease of use is provided by their web-based nature, using upcoming vendor-independent, open standards like HTML5 and WebGL. The applicability of new clients such as Layar are investigated (Figure 2, left) 3D visualisation of the TUD campus in Layar (iPhone), overlay of utilities on panoramic images ([18], Figure 2 middle) and serious games (training with real-world 3D models, Figure 2 right).

The developed model and systems architecture will be tested with two scenarios: high-performance quays and underground pipes. Both scenarios have been identified as having critical issues in internal studies of the Port of Rotterdam and other stakeholders such as the City of Rotterdam. A quay wall can be seen as an assembly of structural engineering components, a series of logistical units consisting of bollard slots, a piece of real estate or as the target zone of a navigation channel that has been externally contracted for constant excavation to guarantee a certain ship draft for a client. When frequent reoccurring changes, such as renting out a berth to a new client, have to be organised. Presently this is done in an ad hoc process where relevant information is gathered, processed and communicated in an unstructured way involving different internal and external stakeholders. Since information systems only exist within specialized domains (e.g. infrastructural engineering, infrastructural facility management, financial management) and no interfaces between these systems have been formally defined, a high amount of manual, error-prone work is involved in the current practice. Often objects appear with 

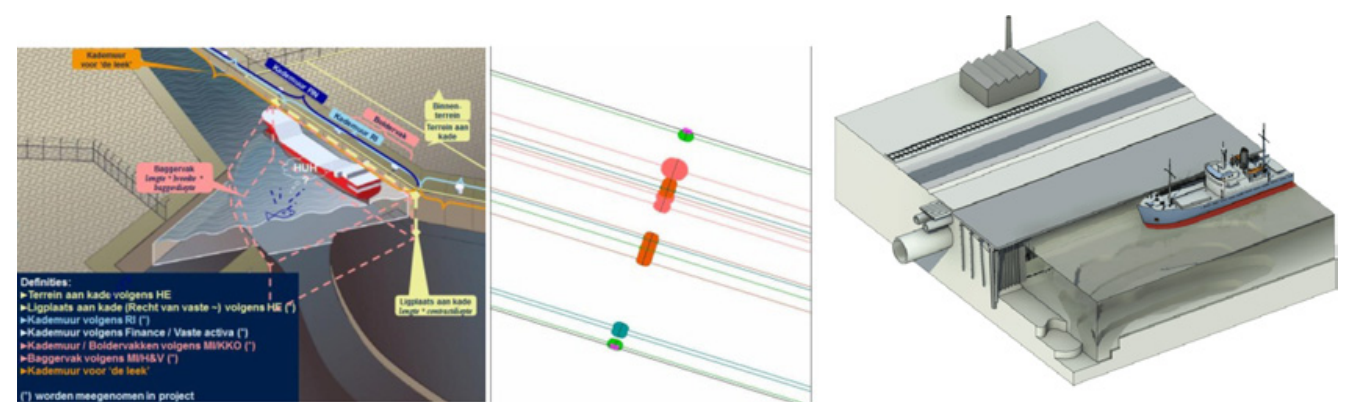

Figure 3. Complex definition of objects for Quays (integrating BIM and GIS) (left) and analysis of utilities (integration of underground information) (middle), intended representation (right).

different definitions by the domain applications (Figure.3, left).This use case is intended for testing the integrated model (especially BIM and GIS integration) and the performance of 3D spatial analysis.

The second use case aims to be used for testing the model and the 3D visualisation aspects of 3DSII. Underground cables and other infrastructural features are obtained from external parties such as the City of Rotterdam in the form of 2D drawings (although the existence of 3D records) that are copied in bulk on a regular, yet informal basis (Figure 3, middle). The planning, execution and documentation of new infrastructural projects however is depending on additional, three dimensional aspects and needs to be streamlined in order to prevent information loss and resulting unwanted side-effects such as cable damage during construction and unnecessary earthworks due to ill coordinated, parallel projects. In contrast Figure 3 right illustrates a possible future representation, which should reflect in the best possible way the real-world phenomena and artefacts.

\section{INITIAL PROTOTYPES}

The first developments concentrate on two aspects related to the use of the model: 3D analysis (clip and profile) and 3D visualisation (Web GL). One of the largest advantages of a well-structured 3D model is the ability to integrated data in one 3D environment and to perform 3D analysis. Therefore as a first step even before developing the model, we considered important to demonstrate some aspects of $3 \mathrm{D}$ visualisation and 3D analysis. A test area in the area of Maasvlakte 2 was identified and all possible data sets (currently in possession or of importance for Port Rotterdam) were collected and made available for testing the developments.

The goal of the 3D visualisation research is to investigate and select an appropriate approach for integrated visualisation of above and underground data in one environment as the focus was on pipes and cables. Utility networks are tricky objects for visualisation because they are usually maintained as simple lines and require significant modifications to be adapted for 3D rendering. Additionally we wanted to investigate the performance of new technologies such as Web GL (X3D/X3DOM and SceneJS) and Layar to create a client-service application for general purpose. The prototype should be able to:

- extract pipes and cables from a data base with their attributes

- convert the lines to an appropriate 3D representation (using cylinders)

- visualise the 3D pipes (using WebGL or Layar) together with above ground information

- allow for simple interaction such as query of attributes, switching layers, etc.

Several prototypes have been developed and tested by now. The final results of all the comparisons are expected after the summer of 2012. At the moment, it has been successfully demonstrated that the needed transformations and adaptations can be executed in the required time to make the work with the model comfortable. Figure 4 illustrates the integrated visualisation of buildings (CityGML LOD2), 3D utility networks and surface data (topographic map of 1:10000). Further investigations concentrate on 


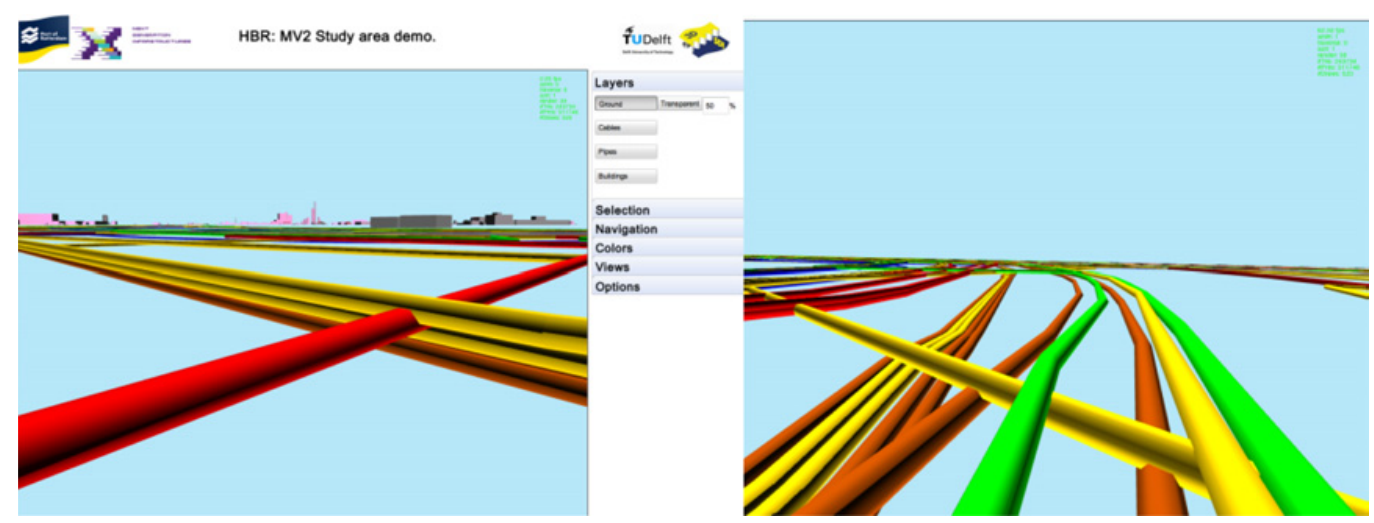

Figure 4. Visualisation of pipes with X3D/X3DOM (courtesy Josafat Guerrero).

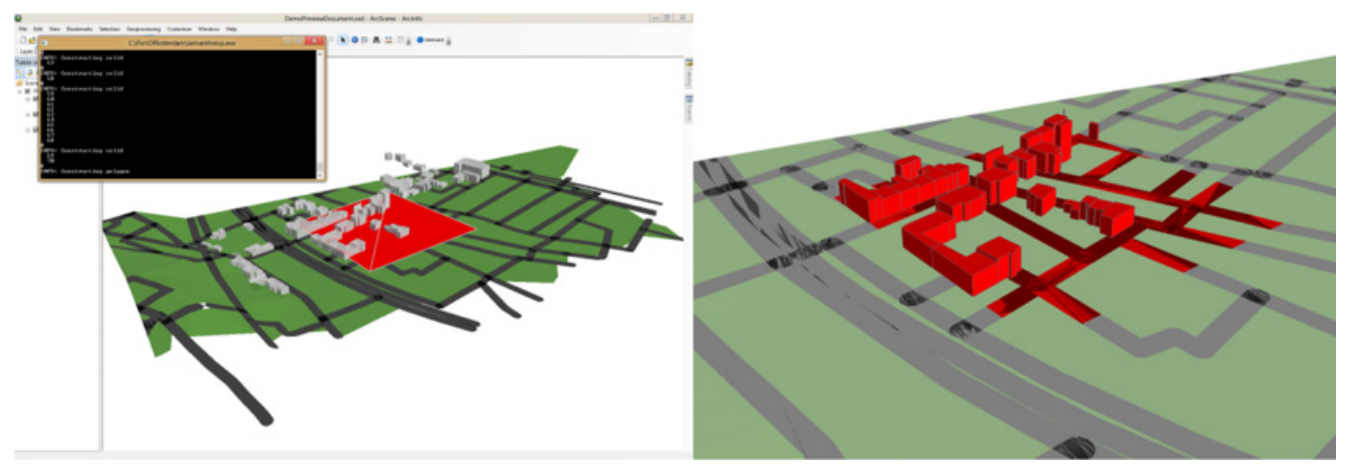

Figure 5. Selection of an area in a 3D model (left) and the corresponding clip of objects inheriting the attributes of the original objects (courtesy Wouter Goedhart).

way the way 3D pipes have to visualised, e.g. the number of faces, which has to be used to represent one cylinder, the max number of objects that should be visualised in one scene, etc.

The goal of the 3D analysis research and developments is to investigate appropriate methods to perform 3D clip, intersections and profiles. Our study on the process management revealed that these operations would be needed in several stages of the project management of a new development (e.g. quay). Firstly, 3D clip operation is needed at the very first stage when a cost-benefit analysis has to be performed. The area for development has to be delineated and analysed what existing objects will be affected. This operation is currently performed in 2D, which often leads to miscomputations and inaccuracies. The clips and the profiles are therefore intended to be in $3 \mathrm{D}$, ensure the validity of object and preserve the attributes of the original object. Figure 5 demonstrates the selection of the area and the 3D clip operation. The profile operation can be performed in all directions (not only vertical), which could be a solution to providing 2D geometries to existing systems, which cannot easily be extended to 3D. Presently the developed algorithms can be applied to CityGML models, but the intention is to be adapted for the model that would be accepted for the Port of Rotterdam.

\section{CONCLUSIONS AND FUTURE DEVELOPMENTS}

In this paper we presented the challenges in spatial information management and the intended developments toward corporate 3DSII for the Port of Rotterdam. Our research concentrates on technical 
aspects of 3D SII, i.e. 3D conceptual model, 3D visualisation and 3D operations to be performed on the model. Appropriate system architecture will be investigated as well to serve the different users and their tasks in a convenient way. Although, the project is in a very initial stage, several important parts of the research have been explored, which resulted in various prototypes. Currently the prototypes are individual components utilising data from files and DBMS (PostGIS). Further developments will address the integration of these components. Shortly after that, the prototypes are going to be presented to and evaluated by specialists of Port Rotterdam in near future.

The most challenging aspect in this research is the conceptual model. The model should serve as a virtual container where all kind of (spatial) objects can be appropriately accommodated for integrated visualisation and analysis. The model might not be physically implemented in any system, but parts of it can be of use in the different departments or sections of the Port. This also implies that many of the current systems will not need to be modified. The internal models will remain untouched and appropriate mappings (semantic and geometric) will be provided. A formal framework for performing the mappings has to be developed as well.

A critical question to be answered is the representation of constructions (as quays, cranes, etc.). New designs of such constructions are increasingly submitted as BIM models. These design models have to be then linked to existing spatial objects, which are mostly maintained following GIS concept. As wellknow from the literature, BIM and GIS concepts for representing features differ significantly. A critical analysis has to be therefore performed to evaluate the applicability of the two concepts and estimate the consequences on the generic model. Several options will be investigated: 1) keep BIM representation as core and map to GIS representation whenever necessary, 2) keep GIS representation and convert the new BIM designs upon receive and 3) keep both representation and establish links between semantically similar objects and their elements. The third approach is being currently investigated since no conversions will be required.

The authors are grateful to the Dutch program NGInfra, which made possible the research within this project. The authors are thankful to all partners in the project from Port Rotterdam and City of Rotterdam for the fruitful discussions and remarkable commitment. The authors also appreciate highly the discussion and exchanged ideas within the COST action TU801, which gave inspiration for the initiation of this project.

\section{References}

[1] K.L. Emgård and S. Zlatanova, Design of an integrated 3D information model, Urban and regional data management: UDMS annual 2007, Taylor and Francis Group, London, 143-156 (2008)

[2] A. Lapierre and P. Cote, Using Open Web Services for urban data management: a testbed resulting from an OGC initiative offering standard CAD/GIS/BIM services, Urban and Regional Data Management; UDMS Annual 2007, Taylor and Francis, London, 381-393 (2008)

[3] F. Penninga and P.J.M. van Oosterom, A simplicial complex-based DBMS approach to 3D topographic data modelling, in: International Journal of Geographical Information Science, 22, 7 , 751-779 (2008)

[4] P. Scarponcini, S. Camateros, O. Custers, S. Zlatanova and P. van Oosterom, Introduction to SII, Creating Spatial Information Infrastructures: Towards the Spatial Semantic Web, CRCpress Taylor and Francis group, Boca Raton, xiii-xxiii (2008)

[5] F. Döner, R. Thompson, J. Stoter, Ch. Lemmen, H. Ploeger, P. van Oosterom and S. Zlatanova, 4D cadastres: First analysis of legal, organizational, and technical impact - with a case study on utility networks, Land Use Policy, 27, 1068-108 (2010) 
Usage, Usability, and Utility of 3D City Models

[6] Stoter, J., M. Reuvers, G. Vosselman, J. Goos, L. van Berlo, S. Zlatanova, E. Verbree and R. Kloosterg, Towards a 3D geo-information standard in the Netherlands, International Archives of the Photogrammetry, Remote sensing and Spatial Information Sciences, XXXVIII-4/W15, November 4-5, 2010, Berlin, Germany, 63-67 (2010)

[7] L. Bodum, E. Kjems, J.Kolar, P. M. Ilsoe, and J.Overby, GRIFINOR: Integrated Object-Oriented Solution for Navigating Real-Time 3D Virtuel Environments, Geo-information for Disaster Management, 937-949 (2005)

[8] G. Gröger, T. Kolbe and A. Czerwinski, OpenGIS CityGML Implementation Specification. http://www.opengeospatial.org/standards/citygml (last accessed 25-07-2012)

[9] P. Coates and Yusuf Arayici, Optimisation of the BIM authoring tool in Architectural practice: a case study approach, Int. J. on 3D modelling, 1, 2, 30-45 (2012)

[10] I. Hijazi, M. Ehler and S. Zlatanova, BIM for geo-analysis (BIM4GEOA): set up of 3D information system with open source software and open specifications (OS), International Archives of the Photogrametry, Remote sensing and Spatial Information Sciences, Vol. XXXVIII4/W15, November 4-5, 2010, Berlin, Germany, 45-49 (2010)

[11] U. Isikdag, and S. Zlatanova, Towards defining a framework for automatic generation of buildings in CityGML using BIM, 3D geo-information sciences, LNG\&C, Springer Verlag, 79-96 (2009)

[12] J. Döllner, and B. Hagedorn, Integrating GIS, CAD and BIM data by service-based virtual 3D city models, Urban and Regional Data Management; UDMS Annual 2007, 157-170 (2008)

[13] J. Beetz, van Berlo, L., de Laat, R. and van den Helm, P. Bimserver.org - an Open Source IFC model server, Proc. of. 27th International Conference on Applications of IT in the AEC Industry CIB-W78, Cairo, November 2010, 1-8 (2010)

[14] C. Hess, C. and M. de Vries, From models to data: A prototype Query Translator for the cadastral domain, Computers, Environment and Urban Systems, 30, 529-542 (2006)

[15] Beetz, J., van Leeuwen, J.P. and de Vries, B, IfcOWL: A case of transforming EXPRESS schemas into ontologies, Artificial Intelligence for Engineering Design, Analysis and Manufacturing. 23, 1, 89-101 (2009)

[16] M.F. Goodchild, Citizens as sensors: the world of volunteered geography, GeoJournal 69, 4, 211221 (2007)

[17] M. Götz and A. Zipf, Towards defining a framework for the automatic derivation of 3D CityGML models from volunteered geographic information, in Int. J. of 3D modelling, 1, 2,1-16 (2012)

[18] E. Verbree, S. Zlatanova and K. Smit, Interactive navigation services through value-added navigation CycloMedia panoramic images, Proceedings of Sixth International Conference on Electronic Commerce, 25-27 October, Delft, The Netherlands, CDROM, 10 p (2004) 